\title{
APLICAÇÃo CATALÍTICA DE PENEIRAS MOLECULARES BÁSICAS MICRO E MESOPOROSAS
}

\author{
Leandro Martins e Dilson Cardoso* \\ 13565-905 São Carlos - SP \\ Recebido em 30/3/05; aceito em 29/6/05; publicado na web em 20/1/06
}

Departamento de Engenharia Química, Universidade Federal de São Carlos, Rodovia Washington Luís, km 235,

\begin{abstract}
CATALYTIC APPLICATIONS OF BASIC MICRO AND MESOPOROUS MOLECULAR SIEVES. Catalysis by solid acids has received much attention due to its importance in petroleum refining and petrochemical processes. Relatively few studies have focused on catalysis by bases and even les on using basic molecular sieves. This paper deals with the potential application of micro and mesoporous molecular sieves in base catalysis reactions. The paper is divided in two parts, the first one dedicated to the design of the catalysts and the second to some relevant examples of catalytic reactions, which find a huge field of applications essentially in the synthesis of fine chemicals. Here, recent developments in catalysis by basic molecular sieves and the perspectives of applications in correlated catalytic processes are described.
\end{abstract}

Keywords: molecular sieves; base catalysis; zeolites.

\section{INTRODUÇÃO}

Em comparação com a vasta aplicação de peneiras moleculares (PMs) ácidas como catalisadores sólidos em processos químicos industriais, pouca atenção tem sido dada àquelas com propriedades básicas ${ }^{1}$. Grande parte do investimento que tem sido empregado nas PMs ácidas é estimulado pelo fato de que as reações químicas envolvidas nos processos petroquímicos (e.g. craqueamento e isomerização) são tipicamente catalisadas por ácidos. Segundo Tanabe e Hölderich ${ }^{2} 127$ processos industriais utilizam catalisadores com propriedades ácidas, sendo que apenas 10 são catalisados por materiais com propriedades básicas. Dentre esses, apenas um exemplo emprega zeólitas básicas: a ZSM-5 impregnada com sulfato de césio (teor de césio 20-25\% em massa), utilizada pelo grupo Merck na produção do 4-metil tiazol (Equação 1), um intermediário na síntese do tiabendazol (fungicida) ${ }^{2}$. A rota industrial anterior de obtenção do 4-metil tiazol envolvia 5 etapas, além do emprego de produtos químicos muito agressivos, tais como os gases cloro e amônia. $\mathrm{O}$ menor número de etapas no novo processo industrial, a estabilidade térmica e capacidade de regeneração dessa PM microporosa permitiu seu emprego na rota atual ${ }^{3}$.<smiles>CN=C(C)C</smiles>

Além deste exemplo, as peneiras moleculares microporosas (particularmente zeólitas) e mesoporosas com propriedades básicas apresentam potencial para aplicação em muitas outras reações de importância comercial. Parte desse potencial está na possibilidade de ajuste das suas propriedades catalíticas, suas atividades, seletividades e estabilidades, através do controle da sua composição química e por tratamentos químicos posteriores às sínteses propriamente ditas ${ }^{4}$.

Em muitos casos, os catalisadores básicos podem ser usados em substituição aos ácidos com ganhos em termos de seletividade, por inibirem a ocorrência de reações paralelas, tais como craqueamento e formação de coque. A utilização de catalisadores heterogêneos bási-

*e-mail: dilsoncardoso@power.ufscar.br cos em reações orgânicas, ao invés de homogêneos, desperta interesse pela redução do número de operações de separação e neutralização envolvidas e pela redução do impacto ambiental decorrente dos rejeitos líquidos $^{5,6}$. Em particular, o emprego de peneiras moleculares pode proporcionar aumento significativo na seletividade à formação de produtos com menor diâmetro cinético, devido a limitações estéricas e difusionais nos poros do catalisador ${ }^{7,8}$. Um exemplo do efeito desta seletividade, denominada como seletividade de forma, é a adição cíclica do dióxido de carbono a epóxidos ${ }^{9,10}$. Esta reação gera produtos que apresentam grande variedade de aplicações despertando, conseqüentemente, o interesse industrial ${ }^{11}$.

O objetivo deste trabalho é examinar uma das faces catalíticas das zeólitas menos exploradas na literatura, que provou ser muito valiosa na síntese de intermediários químicos e na química fina. Deve ser ressaltado, no entanto, que o diâmetro cinético dos compostos gerados nestas reações está limitado pelo diâmetro dos microporos das zeólitas, os quais se encontram geralmente na ordem de até 0,75 $\mathrm{nm}$. Com a descoberta de materiais com poros e canais altamente ordenados, com diâmetros na faixa dos mesoporos ${ }^{12,13}$, a perspectiva de aplicação destas peneiras moleculares no processamento de moléculas com diâmetro na faixa de 2-50 nm tem aumentado continuamente. Desta forma, algumas das reações discutidas neste artigo podem se tornar ainda mais valiosas quando o conhecimento desse novo material com propriedades básicas estiver consolidado.

\section{BASICIDADE DE ALUMINO-SILICATOS MICROPOROSOS}

Os alumino-silicatos podem ser amorfos ou ordenados e, estes últimos, ter uma estrutura micro ou mesoporosa. Particularmente, os alumino-silicatos cristalinos microporosos são denominados zeólitas. A estrutura cristalina deste grupo de materiais é constituída pela combinação tridimensional de tetraedros do tipo $\mathrm{TO}_{4}^{-}$, onde $\mathrm{T}$ representa os átomos de silício ou alumínio, unidos entre si através de átomos de oxigênio comuns ${ }^{7,8}$. Sendo o alumínio trivalente, os tetraedros $\mathrm{AlO}_{4}{ }_{4}$ induzem cargas negativas na estrutura, as quais são neutralizadas por cátions de compensação (Esquema 1). Estes cátions, juntamente com as moléculas de água, outros adsorbatos e sais, estão localizados nos canais e cavidades desta estrutura ${ }^{7}$. Este tipo de estrutura microporosa 
faz com que as zeólitas apresentem elevada área superficial e se diferenciem de outros sólidos porosos por impedir o acesso, ao seu interior, de moléculas maiores que a dimensão de seus poros, daí a propriedade que originou o termo peneira molecular.

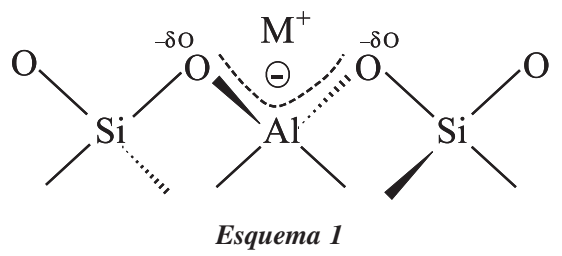

As características ácidas das zeólitas estão associadas a dois tipos de sítios: a átomos de alumínio tetracoordenados, pertencentes à estrutura cristalina, cuja carga negativa é compensada por cátions e, particularmente, por prótons, resultando em acidez de Brönsted e, a átomos de alumínio com coordenação octaédrica localizados fora da rede, gerando acidez de Lewis.

Por sua vez, os sítios básicos em zeólitas estão associados aos ânions oxigênio pertencentes à rede ${ }^{14,15}$. Neste caso, por serem intrínsecos (ou estruturais), o número de sítios básicos é igual ao número de átomos de alumínio na estrutura. A força desses sítios depende, entre outros fatores, da acidez do cátion $\mathrm{M}^{+}$neutralizando a carga do oxigênio (Esquema 1).

A existência de pares conjugados foi observada nas zeólitas primeiramente por Barthomeuf ${ }^{16}$ que, comparando medidas de acidez e basicidade, verificou que zeólitas do tipo faujasita (FAU) apresentam sítios ácido-básicos conjugados, sendo que a força de um aumenta quando a do outro diminui. Portanto, no Esquema 1, quanto menor a acidez do cátion $\mathrm{M}^{+}$, maior a basicidade do ânion oxigênio. A acidez do cátion $\mathrm{M}^{+}$, por sua vez, diminui quando seu raio é aumentado, distribuindo sua carga positiva em um volume maior (menos eletronegativo). Por ex., para metais alcalinos presentes em zeólitas a basicidade do oxigênio de rede aumenta na seguinte ordem $\mathrm{Li}<\mathrm{Na}<\mathrm{K}<\mathrm{Rb}<\mathrm{Cs}^{17}$. Quando a densidade de ânions tetraédricos $\mathrm{TO}_{4}^{-}$(Esquema $1, \mathrm{AlO}_{4}^{-}$) é aumentada na rede cristalina da zeólita, além do aumento no número de sítios básicos, a basicidade também é maior.

A basicidade teórica de uma zeólita pode ser calculada utilizando-se o princípio da equalização de eletronegatividade de Sanderson. Esta metodologia foi aplicada pela primeira vez em zeólitas por Mortier $^{18}$. Para um composto qualquer com fórmula genérica $P_{p} Q_{q} R_{r}$, a eletronegatividade intermediária $\left(\mathrm{S}_{\text {int }}\right)$ é obtida pela Equação 2 , onde $\mathrm{P}, \mathrm{Q}$ e $\mathrm{R}$ são elementos químicos e $\mathrm{S}_{\mathrm{j}}$ é a eletronegatividade do átomo $\boldsymbol{j}$. Já a Equação 3 fornece a carga parcial localizada no átomo de oxigênio. Quanto maior for a basicidade de Lewis do oxigênio, maior será o valor de $-\delta_{\text {Oxigênio }}$. Na Figura 1 é mostrado que a carga negativa $-\delta_{\text {Oxigenno }}$ aumenta quando o teor de alumínio tetracordenado e o tamanho do cátion de compensação aumentam $\left(\mathrm{M}^{+}\right.$no Esquema 1), isto é, quando a eletronegatividade do cátion diminui ${ }^{18,19}$.

$$
\begin{aligned}
& S_{\text {int }}=\left[S_{P}^{P} \cdot S_{Q}^{q} \cdot S_{R}^{r}\right]^{1 /(p+q+r)} \\
& -\delta_{\text {oxigênio }}=\frac{\left(S_{\text {int }}-S_{\text {oxigênio }}\right)}{2,08 \cdot\left(S_{\text {oxigênio }}\right) 1 / 2}
\end{aligned}
$$

Apesar de os resultados obtidos com os cálculos teóricos da carga parcial localizada sobre os átomos de oxigênio $\left(-\delta_{\text {Oxigênio }}\right)$ apresentarem boas correlações com os resultados experimentais ${ }^{16,17,20}$, outros parâmetros podem afetar as cargas e sua distribuição na estrutura. Experimentos de adsorção $0^{21}$ e catálise ${ }^{22}$ mostraram que algumas estruturas zeolíticas, em particular a mordenita e

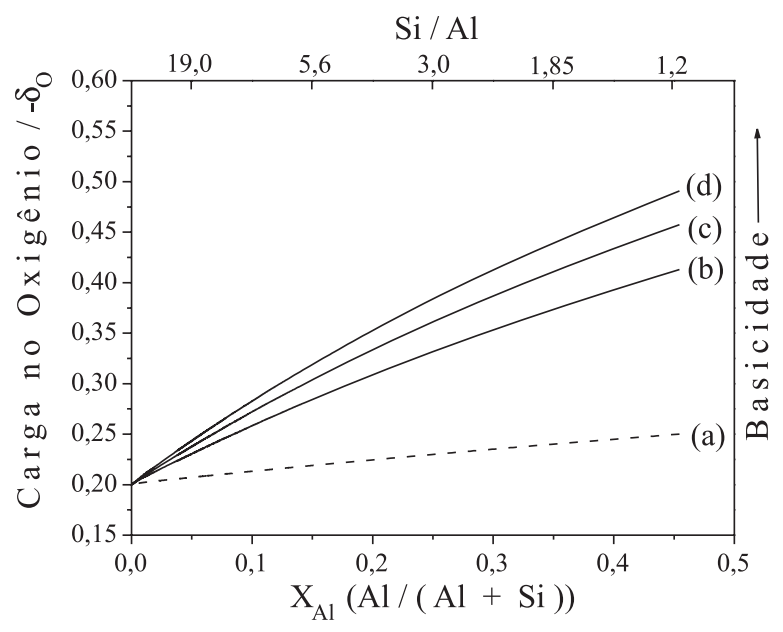

Figura 1. Influência do teor de alumínio sobre a carga teórica localizada sobre os átomos de oxigênio $\left(-\delta_{\text {oxiênio }}\right)$ para zeólitas protônicas $(a)$ ou contendo $\mathrm{Na}(b), K(c)$ e Cs (d). Adaptada da ref. 19

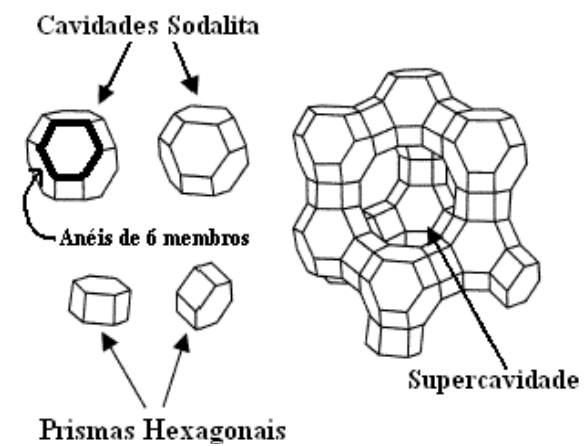

Figura 2. Diagrama esquemático de uma zeólita com estrutura faujasita. À esquerda: unidades de construção. À direita: estrutura faujasita

a beta, apresentam uma basicidade maior que a calculada. Similarmente, sugere-se que a peneira molecular MCM-22 trocada com metais alcalinos, apesar da baixa quantidade de alumínio presente no sólido, apresente propriedades básicas ${ }^{23}$. Estas observações indicam que, além da quantidade de $\mathrm{Al}$ e da eletronegatividade do cátion, outros fatores também controlam a basicidade dessas peneiras moleculares. Por ex., estruturas zeolíticas distintas induzem diferentes ângulos e comprimentos de ligações. Recentemente foi mostrado que a basicidade dos átomos de oxigênio presentes na estrutura FAU é maior quando dois átomos de $\mathrm{Al}$, presentes em um anel de 6 membros (mostrado na Figura 2), se encontram em posição meta ou quando dois átomos de $\mathrm{Al}$ se encontram em um anel de 4 membros. A basicidade é diminuída quando dois átomos de alumínio se encontram em posição para, do anel de 6 membros ${ }^{24}$. Portanto, a influência da estrutura da PM na localização dos átomos de alumínio e, conseqüentemente, na sua basicidade, também deve ser considerada quando estruturas distintas são comparadas.

Na Tabela 1 são mostradas as características dos poros e a menor razão molar $\mathrm{Si} / \mathrm{Al}$ encontrada em algumas peneiras moleculares. Como já discutido, a incorporação máxima de átomos de Al na rede de uma determinada estrutura, gerando conseqüentemente uma máxima basicidade, depende de sua topologia. Por ex., a estrutura MFI permite uma incorporação de átomos de alumínio em sua estrutura tal que a menor razão $\mathrm{Si} / \mathrm{Al}$ encontrada é em torno de 11. No entanto, a despeito do baixo teor de alumínio presente nessa estrutura, para catálise básica, foi verificada a presença de espécies $\mathrm{AlO}-(\mathrm{SiO})_{\mathrm{x}}-\mathrm{AlO}(\operatorname{com} \mathrm{x}=1 \mathrm{e}$ 
Tabela 1. Características dos poros e menor razão molar Si/Al encontrada em algumas peneiras moleculares ${ }^{28}$

\begin{tabular}{lcccc}
\hline Tamanho do poro & Diâmetro do poro $(\mathrm{nm})$ & Nome comum & Símbolo estrutural & Menor razão Si/Al \\
\hline Pequeno & 0,41 & Zeólita A & LTA & 1 \\
Médio & $0,53 \times 0,56$ & ZSM-5 & MFI & 11 \\
Grande & 0,74 & Zeólita X & FAU & 1,5 \\
& $\sim 0,6 \times \sim 0,7$ & Zeólita $\beta$ & BEA & 6 \\
Mesoporoso & $1,5-10$ & MCM-41 & $\ldots$ & $\ldots$ \\
\hline
\end{tabular}

2), ou seja, regiões com alta densidade de $\mathrm{Al}$, garantindo a observação experimental de basicidade ${ }^{25,26}$. Dependendo das condições de síntese da estrutura MFI com uma razão $\mathrm{Si} / \mathrm{Al}=12,5$, pode-se obter de 40 a $80 \%$ dos átomos de alumínio formando predominantemente aglomerados do tipo $\mathrm{AlO}-(\mathrm{SiO})_{2}-\mathrm{AlO}^{25,27}$. Já para a estrutura FAU, a possibilidade de incorporação de um alto teor de alumínio $(\mathrm{Si} / \mathrm{Al}=1,5)$ e o maior diâmetro de poros (acessível a um maior número de moléculas) tornam esta zeólita uma PM alvo de muitos estudos em catálise básica.

Uma outra alternativa na obtenção de peneiras moleculares microporosas com propriedades básicas é através da impregnação destes materiais, que neste caso funcionam como suportes, com compostos que possuem propriedades básicas. Uma das primeiras tentativas na obtenção destes catalisadores básicos, descrita por Martens et al. $^{29}$, envolveu a dispersão de agregados de sódio nos poros da zeólita faujasita. De acordo com este pesquisador, o melhor procedimento para obtenção dessas partículas básicas é através da decomposição da azida de sódio dispersa na zeólita desidratada. Este procedimento conduz à formação de sítios básicos muito fortes, que são altamente ativos para uma grande variedade de reações orgânicas ${ }^{29}$. Entretanto, o grande problema com este tipo de catalisador reside na alta sensibilidade à exposição dos sítios ativos ao ar. As partículas de sódio reagem com a umidade quando expostas ao ar, ocorrendo, conseqüientemente, perda de atividade.

Como a basicidade intrínseca à estrutura das zeólitas apresenta força mediana, a melhora desta propriedade é de grande interesse. Um método promissor, desenvolvido por Hathaway e Davis ${ }^{30}$, permitiu criar um catalisador com sítios mais estáveis à atmosfera oxidante, que no caso dos agregados de sódio. Este método dispersa partículas de óxido de césio nos canais da zeólita, pela impregnação da zeólita $\mathrm{NaY}$ com acetato de césio. Em seguida, o acetato pode ser decomposto por calcinação em ar a $400-450{ }^{\circ} \mathrm{C}^{31}$. Se uma temperatura maior for empregada, a formação de Cs metálico é favorecida $\left(2 \mathrm{Cs}_{2} \mathrm{O} \rightarrow \mathrm{CsO}_{2}+3 \mathrm{Cs}\right)^{32}$. Como o dióxido de césio $\left(\mathrm{CsO}_{2}\right)$ é volátil a uma temperatura maior que $500{ }^{\circ} \mathrm{C}$, o catalisador torna-se enriquecido com agregados de césio, que é catalisador básico por si próprio ${ }^{32}$. O mesmo conceito envolvido na preparação deste tipo de catalisador foi empregado na obtenção da única zeólita com propriedades básicas aplicada atualmente em um processo industrial: a ZSM-5 impregnada com sulfato de césio ${ }^{2}$.

\section{BASICIDADE DE ALUMINO-SILICATOS MESOPOROSOS}

Além da demanda no processamento de moléculas que possuam diâmetro cinético mais volumosas (superior a $0,75 \mathrm{~nm}$ ), o desenvolvimento de catalisadores com alta área específica, ou com alta dispersão da área ativa, é uma necessidade imprescindível na melhoria do desempenho dos atuais processos industriais ${ }^{28}$, pois há uma melhoria no transporte de massa de reagentes e produtos. A descoberta recente de materiais com mesoporos altamente ordenados, ou seja, com diâmetro entre 2 e 50 nm (Figura 3), e alta área superficial ${ }^{12,13}$, de até $1600 \mathrm{~m}^{2} \mathrm{~g}^{-1}$, introduziu novas perspectivas no desenvolvimento de catalisadores mais eficientes ${ }^{33,34}$. Diferentemen- te das zeólitas, esses materiais apresentam paredes amorfas, já que os átomos que constituem o esqueleto inorgânico não possuem uma distribuição espacial regular ${ }^{33}$.

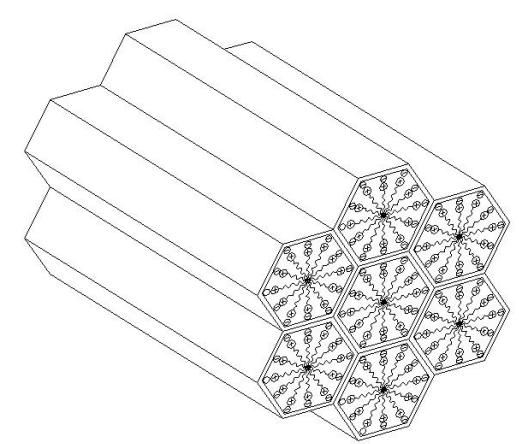

Figura 3. Sólido mesoporoso MCM-41 com poros preenchidos com o agente direcionador $\mathrm{C}_{16} \mathrm{H}_{33}\left(\mathrm{CH}_{3}\right) \mathrm{N}^{+}$

No contexto atual das peneiras moleculares mesoporosas, três materiais, obtidos por métodos diferentes de síntese, vêm sendo preferencialmente estudados. O primeiro, faz parte da família de PMs denominadas M41S, descoberto pela empresa Mobil ${ }^{12,13}$, e composto basicamente por silício ou silício e alumínio. Esta família incluí a PM com arranjo hexagonal MCM-41 (mostrada na Figura 3), a MCM48, que possui organização cúbica, e a lamelar MCM-50. A preparação desses materiais da família M41S envolve o uso de um surfactante, exemplificado pelo brometo de cetiltrimetilamônio $\left(\mathrm{C}_{16} \mathrm{H}_{33}\left(\mathrm{CH}_{3}\right) \mathrm{N}^{+} \mathrm{Br}\right.$ ou genericamente $\mathrm{CTABr}$ ), como agente direcionador de estrutura, e uma fonte de silício e alumínio.

O segundo material introduzido por Pinnavaia et $a l^{35,36}$ foi obtido na presença de aminas neutras ( $e$. $g$. hexadecilamina) ou de surfactantes neutros à base de óxido de polietileno, resultando nas estruturas HMS e MSU, respectivamente. Essas estruturas apresentam canais cilíndricos, porém estes não são paralelos como a MCM-41, e sim na forma de "caminhos de minhoca" ("wormholelike pore").

Finalmente, Stucky et al. ${ }^{37}$ introduziram uma nova rota de síntese para materiais mesoporosos envolvendo co-polímeros tribloco como agentes direcionadores de estrutura. Estes materiais, exemplificados pela SBA-15, apresentam mesoporos também ordenados e monodirecionais, mas com diâmetros maiores que 50 $\mathrm{nm}$ e paredes mais espessas que a MCM-41. Isto a torna mais estável termicamente que os materiais descritos anteriormente.

Assim como ocorre com as peneiras moleculares microporosas, a catálise básica empregando as mesoporosas tem sido menos discutida que a catálise ácida. Entretanto, alguns mostram que PMs mesoporosas apresentam potencial para serem utilizadas na catálise básica. Os sítios básicos presentes nestes materiais podem se apresentar de 4 formas possíveis: basicidade intrínseca, gerada por elementos trivalentes, pertencentes à rede, como por ex. o Al, que pode ser controlada através de procedimentos de troca-iônica ${ }^{37-39}$; óxidos metálicos com alto caráter básico dispersos nos canais ${ }^{40,41}$; grupos $\mathrm{SiO}^{-}$terminais, de alta basicidade, pertencentes à estrutura 
e presentes na superfície externa ou nos canais ${ }^{42-44} \mathrm{e}$, compostos orgânicos ligados à rede, contendo grupos funcionais com propriedades básicas (funcionalização) ${ }^{45-47}$

Da mesma maneira como discutido para as zeólitas, a incorporação de alumínio na estrutura da PM mesoporosa induz uma carga negativa, que gera acidez quando compensada por um próton. Através de um procedimento simples de troca-iônica com metais alcalinos obtém-se a primeira forma de basicidade, acima indicada. Dependendo da acidez do cátion é possível, em princípio, obter PMs mesoporosas com átomos de oxigênio reticulares mais ou menos ácidos. Bonelli et al. ${ }^{38}$ prepararam uma amostra de [Si,Al]MCM-41, na forma sódica e com razão $\mathrm{Si} / \mathrm{Al}=20$ e, após um procedimento de troca-iônica em meio aquoso, obtiveram amostras contendo $\mathrm{Cs}^{+}$e $\mathrm{K}^{+}$. Apesar de medidas de composição química indicarem a troca de quase $100 \%$ do sódio presente por césio ou por potássio, experimentos de adsorção de $\mathrm{CO}_{2}$ não evidenciaram a presença de sítios básicos. A ausência de basicidade, neste caso, está relacionada com a baixa incorporação de alumínio na estrutura dessa peneira molecular (Figura 1). A zeólita ZSM-5, trocada com vários metais alcalinos e com razão $\mathrm{Si} / \mathrm{Al}$ similar, também não evidenciou a presença de basicidade quando se empregou pirrol adsorvido como molécula sonda ${ }^{22}$.

De forma a desenvolver a basicidade intrínseca desses materiais, já foram realizados vários estudos objetivando aumentar a incorporação de alumínio ${ }^{51-53}$, no entanto, pouco sucesso foi obtido. O problema é que, ao diminuir a razão $\mathrm{Si} / \mathrm{Al}$ para valores menores que 10 ocorreu uma redução significativa da organização mesoporosa $^{51}$. Em algumas situações, para razões Si/Al menores que 2 a difração de raios-X não identificou nenhum arranjo ordenado $^{52}$. Em outros casos, amostras com alto teor de Al mostraram sinais no espectro de $\mathrm{RMN}$ do ${ }^{27} \mathrm{Al}$ característicos de espécies em coordenação octaédrica, como resultado de uma desaluminização parcial das paredes amorfas ${ }^{53,54}$. Mas, segundo trabalhos recentes, é possível obter ${ }^{55,56}$ a MCM-41 bem organizada com razão $\mathrm{Si} / \mathrm{Al}$ em torno de 1,2, utilizando-se altas razões $\mathrm{H}_{2} \mathrm{O} / \mathrm{SiO}_{2}$ e $\mathrm{CTA} / \mathrm{SiO}_{2}$. Estes trabalhos foram publicados há pouco tempo e as propriedades catalíticas destes materiais ainda não foram avaliadas.

A dispersão de óxidos de metais alcalinos nos canais das peneiras moleculares mesoporosas é a segunda forma de gerar sítios básicos. Kloetstra et al. ${ }^{39}$ inicialmente propuseram dispersar partículas de óxido de césio nos canais da MCM-41. Para a preparação, a PM é impregnada com uma solução concentrada de acetato de césio seguida de calcinação para decomposição do íon acetato. Os sítios básicos gerados foram avaliados por dessorção programada de $\mathrm{CO}_{2}{ }^{57}$ e apresentaram maior basicidade que os das amostras só trocadas com Cs. No entanto, devido aos tratamentos sofridos durante a preparação, sua estrutura foi drasticamente afetada, como observado por difração de raios-X e por isotermas ${ }^{39}$ de adsorção/dessorção de $\mathrm{N}_{2}$. É bem conhecido que ligações $\mathrm{Si}-\mathrm{O}-\mathrm{Si}$, presentes nesses materiais, se hidrolizam em altos valores de $\mathrm{pH}$. Como as $\mathrm{PM}$ mesoporosas apresentam uma alta área superficial, a hidrólise dessas ligações é facilitada mesmo em um meio levemente básico. Resultados mostram que um $\mathrm{pH}$ de 8,5 é o valor limite para evitar que a estrutura mesoporosa MCM-41 sofra destruição significativa ${ }^{40}$. O próprio óxido de césio também contribui para a desorganização, através da reação de mineralização ${ }^{38}: \mathrm{Si}-\mathrm{O}-\mathrm{Si}+\mathrm{Cs}_{2} \mathrm{O} \rightarrow 2 \mathrm{SiO}^{-} \mathrm{Cs}^{+}$. Uma forma de resolver esta instabilidade seria empregar materiais mesoporosos com paredes mais espessas e, conseqüentemente, mais estáveis frente a altos valores de $\mathrm{pH}$ e à presença de óxidos básicos. Uma forte candidata à preparação deste catalisador é a peneira molecular SBA$15^{41}$. A dispersão de $\mathrm{MgO}$ através da decomposição de acetato de magnésio não causou mudanças no seu padrão de difração de raios$\mathrm{X}$, indicando a maior estabilidade desta estrutura para a dispersão de óxidos básicos, quando comparada com a MCM-41. Observou-se, no entanto, perda de área específica à medida que se aumenta o teor de $\mathrm{MgO}^{41}$, devido ao bloqueio parcial dos poros.

Em outro trabalho também recente, Kubota et al. ${ }^{42-44}$ obtiveram bons resultados com a MCM-41, gerando sítios básicos através da terceira forma acima indicada, sugerindo seu potencial de aplicação na catálise básica: os autores utilizaram o material mesoporoso $\mathrm{CTA}^{+}$-[Si]-MCM-41, ou seja, com os poros ainda preenchidos pelo surfactante iônico $\mathrm{C}_{16} \mathrm{H}_{33}\left(\mathrm{CH}_{3}\right) \mathrm{N}^{+}$(Figura 3). Ao empregar este catalisador na condensação de Knoevenagel, reação que envolve uma etapa de abstração protônica pelos sítios básicos, foram obtidas altas conversões à temperatura ambiente. Este resultado apresenta uma melhoria significativa em comparação aos resultados obtidos à temperatura de $80{ }^{\circ} \mathrm{C}$, normalmente utilizada para esta reação quando se emprega zeólitas básicas: faujasitas com baixa razão $\mathrm{Si} / \mathrm{Al}$ e contendo $\mathrm{Cs}^{17}$. O emprego de baixas temperaturas é muito importante, não só pela economia de energia nos processos industriais, mas também pela eliminação de subprodutos, como os decorrentes da reação subseqüente de adição de Michael ${ }^{57}$.

Resultados de RMN de ${ }^{29} \mathrm{Si}$ da [Si]-MCM-41 com os poros preenchidos com $\mathrm{CTA}^{+}$indicaram a presença de grande quantidade de arranjos $\left(\mathrm{SiO}_{3}\right) \mathrm{SiO}^{-}$(Figura 4 , onde $\mathrm{M}^{+}=\mathrm{CTA}^{+}$) na sua superfície. Uma vez que o material mesoporoso não continha óxidos metálicos ou resíduos de aminas, os resultados de catálise sugerem que os sítios ativos sejam os ânions $\mathrm{SiO}^{-}$terminais, de alta basicidade ${ }^{44}$. Considerando que os mesoporos ainda continham o direcionador de estrutura, $\mathrm{CTA}^{+}$, conclui-se que a atividade catalítica observada deva-se aos grupos $\mathrm{SiO}^{-}$localizados na entrada dos poros. Diferentemente de hidróxidos ou alcóxidos metálicos, o cátion orgânico $\mathrm{CTA}^{+}$apresenta baixa mobilidade no meio reacional e está localizado próximo ao sítio $\mathrm{SiO}^{-}$da estrutura do material mesoporoso. O fato do cátion $\mathrm{CTA}^{+}$ser grande, faz com que haja menor interação com o sítio SiO, deixando este ânion, que apresenta alto caráter básico, mais disponível para a reação catalítica. Os resultados de catálise mostraram, também, que, ao se utilizar novamente o mesmo catalisador, sua atividade diminui a cada nova reação. Este comportamento revela que durante a reação ocorre a formação de grupos $\left(\mathrm{SiO}_{3}\right) \mathrm{SiOH}\left(\mathrm{M}^{+}=\right.$ $\mathrm{H}^{+}$na Figura 4) mais estáveis, que são responsáveis pela diminuição da basicidade do catalisador.

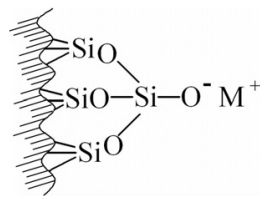

Figura 4. Arranjo ( $\left.\mathrm{SiO}_{3}\right) \mathrm{SiO}^{-}$superficial do material mesoporoso

A quarta e última forma de se obter sítios básicos em peneiras moleculares mesoporosas foi apresentada primeiramente por Brunel et $a l .{ }^{46}$. Eles funcionalizaram a superfície de sólidos mesoporosos com alcoxisilanos, particularmente com aqueles contendo aminas terminais em sua composição $\left(\mathrm{X}=\mathrm{NH}_{2}\right.$, Figura 5). Antes da

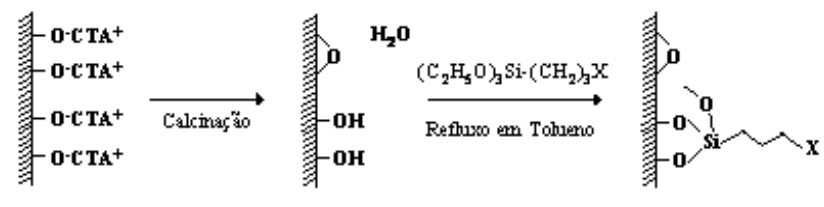

(a) (c) mesoporosa. (a) contendo o direcionador de estrutura orgânico, (b) superfície com condensação parcial de grupos silanóis SiOH e (c) superfície funcionalizada 
funcionalização, o surfactante iônico presente nos poros é removido por calcinação, gerando grupos silanóis, que são os pontos de ancoragem $^{45}$. A concentração dos grupos silanóis depende de como o surfactante iônico é removido. As sílicas mesoporosas calcinadas apresentam menor concentração de grupos silanóis, devido às condensações a altas temperaturas (etapa b, Figura 5). Se o surfactante for removido por extração com solvente, a superfície dos poros se mantém rica em grupos silanóis, com quase $100 \%$ de cobertura $^{48,49}$.

\section{REAÇÕES CATALISADAS POR PENEIRAS MOLECULARES BÁSICAS}

Várias técnicas vêm sendo utilizadas para caracterizar adequadamente os sítios básicos presentes em catalisadores sólidos e, em especial, nas peneiras moleculares ${ }^{4,59}$. Dentre elas destacam-se o uso de moléculas sonda, cuja interação com o sítio básico é acompanhada por técnicas espectroscópicas (i. e. deslocamento de picos ou bandas na região correspondente à ligação química estudada); adsorção ou dessorção à temperatura programada ${ }^{60}$; titulação com indicadores específicos ${ }^{61}$ e, uso de reações modelo ${ }^{4}$.

Apesar da variedade, não existe uma técnica que possa ser empregada de forma geral, pois muitas delas apresentam limitações. Mas, dentre as listadas, a mais atraente é aquela que faz uso de reações modelo. Ao mesmo tempo em que as propriedades básicas do material são avaliadas, o potencial de aplicação do catalisador básico também é evidenciado.

As reações utilizadas para caracterização das propriedades ácido-básicas dos catalisadores devem apresentar características particulares, como por ex., apresentar uma rota reacional conhecida. Desta forma é possível identificar a função de cada sítio (ácido ou básico) na formação dos produtos. Dentre as reações que podem ser utilizadas na caracterização da basicidade de sólidos estão a condensação aldólica ${ }^{61-66}$, a condensação de Knoevenagel ${ }^{8,31,32,47,67,68}$ e a alquilação lateral de aromáticos ${ }^{30,69-72}$.

\section{Condensação aldólica}

As reações de condensação aldólica ${ }^{62-64}$ (Figura 6) são características de aldeídos e cetonas e podem ser catalisadas por sítios ácidos ou básicos para formar $\beta$-hidróxi-aldeídos ou cetonas como produtos primários. As etapas catalíticas subseqüentes dependem da natureza dos sítios ativos. A condensação aldólica da acetona (Composto 1, Figura 6) produz como produto primário, independente do tipo de sítio catalítico, a diacetona álcool (2). Sobre sítios ácidos, esta se decompõe a isobuteno (3) e ácido acético (4). Este último pode se dessorver

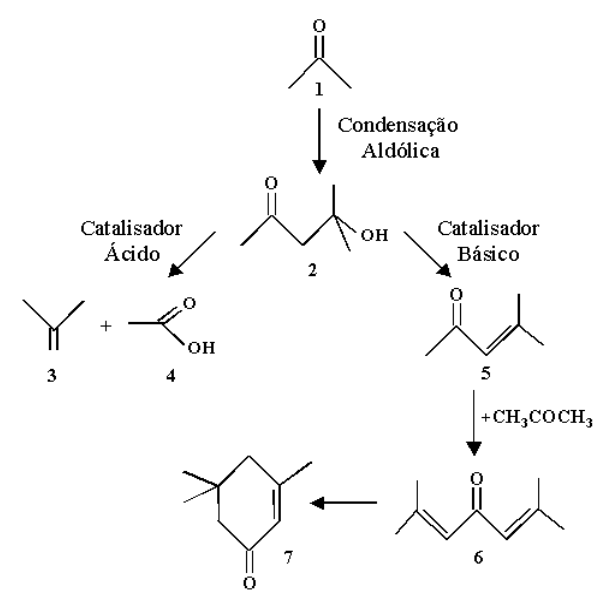

Figura 6. Alguns produtos secundários formados a partir da condensação da acetona ou se decompor em metano e dióxido de carbono, enquanto que o isobuteno se dessorve como produto final ou segue para reações consecutivas de polimerização, isomerização e/ou quebra sobre sítios ácidos. Sobre catalisadores básicos, a condensação aldólica da acetona fornece um conjunto completamente diferente de produtos. A diacetona álcool (2) desidrata-se rapidamente para formar óxido de mesitileno (5), que se dessorve como produto final ou segue para outras reações. As próximas etapas catalíticas incluem a reação do óxido de mesitileno com acetona, formando um intermediário (6) que se rearranja para formar a isoforona (7). Portanto, a condensação aldólica da acetona sobre catalisadores básicos conduz a uma mistura de óxido de mesitileno e isoforona como os principais produtos. Industrialmente, o óxido de mesitileno é hidrogenado seletivamente à metilisobutilcetona, empregando-se um catalisador de paládio suportado em alumina ${ }^{73}$. Este último produto encontra diversas aplicações como solvente de tintas e como agente extrator ${ }^{73}$.

A energia livre de Gibbs padrão da reação de condensação $(\mathbf{1} \rightarrow \mathbf{2})$ é positiva $(\Delta \mathrm{G} \sim 45 \mathrm{~kJ} / \mathrm{mol})$, portanto, a reação exige condições rigorosas, tanto no que se refere à temperatura como à basicidade. A condensação aldólica da acetona sobre as zeólitas A e $\mathrm{X}$ exige, por ex., a dispersão de metais alcalinos no interior de seus poros, o emprego de meio reacional rigorosamente anidro e temperaturas da ordem de $350{ }^{\circ} \mathrm{C}$. Nestas condições são formados óxido de mesitileno e isoforona como os principais produtos $(80 \%$ de seletividade a uma conversão de $8-15 \%)^{64}$. A razão molar entre os dois produtos é dependente do tipo de zeólita empregada. A zeólita $\mathrm{X}$, de maior diâmetro de poros (Tabela 1), favorece a formação da isoforona. Já com a zeólita do tipo A, o óxido de mesitileno, por ser uma molécula menor, é formado preferencialmente. Este comportamento evidencia claramente o efeito da seletividade de forma da zeólita A na formação da molécula com menor diâmetro cinético, em detrimento daquela mais volumosa ${ }^{58}$.

\section{Condensação de Knoevenagel}

A reação de condensação, como visto acima, representa uma classe de reações muito importantes na síntese de compostos intermediários. Moléculas complexas e contendo grupos funcionais específicos podem ser formadas através da condensação de duas ou mais moléculas simples. Dentro dessa classe, a condensação de Knoevenagel (Equação 4) tem sido muito utilizada comercialmente para preparação de intermediários para a química fina e como reação modelo para caracterização da basicidade de catalisadores sólidos ${ }^{1}$. Esta reação ocorre entre um composto carbonílico (aldeídos ou cetonas, composto $\mathbf{1}$, na Equação 4) e moléculas contendo grupos metilênicos ativados (composto 2 ) formando olefinas de alto peso molecular (composto 3 ), que apresentam características muito valiosas nas indústrias farmacêutica e de aromas $^{31}$. Uma importante vantagem desta reação em fase líquida é a possibilidade do uso de reagentes com caráter básico diferenciado. Convencionalmente, esta reação é catalisada por bases fracas, tal como aminas, em meio homogêneo.

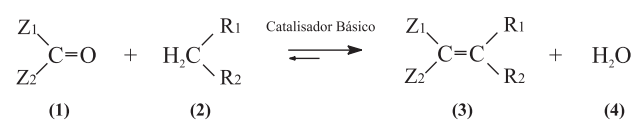

A primeira etapa na condensação é a formação de um carbânion a partir do composto metilênico, pela abstração de um próton pelo sítio básico do catalisador (Equação 5).

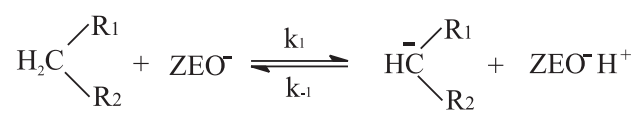


O carbânion então ataca o átomo de carbono do grupo carbonílico, formando uma ligação C-C (Equação 6).<smiles>[R]C=[C+]=CC([Z4])=O</smiles>

Finalmente, ocorre a eliminação de uma molécula de água e a formação de uma ligação $\mathrm{C}=\mathrm{C}$ (Equação 7). Nesta etapa o catalisador é recuperado.

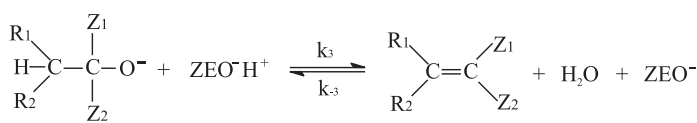

Como já mencionado, a importância do uso de peneiras moleculares com propriedades básicas reside, principalmente, no efeito de seletividade de forma que esses catalisadores proporcionam durante a reação. Em trabalho empregando zeólitas trocadas com cátions alcalinos, Corma et. al..$^{71}$ relataram o efeito da seletividade de forma na condensação do benzaldeído com cianoacetato de etila. As zeólitas apresentam atividade na condensação de Knoevenagel, mas, devido à limitação de espaço nas suas cavidades, ao contrário dos demais sólidos básicos, o produto formado não é consumido na reação série-paralela, de adição de Michael (Equação 8).

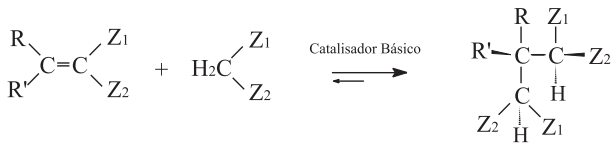

(3)
Kubota et al. $^{44}$, em trabalho recente, mostraram que a peneira molecular $\mathrm{CTA}^{+}$-[Si]-MCM-41, apesar de estar com os mesoporos preenchidos com o tensoativo, é um catalisador com propriedades básicas, promissor para aplicação na síntese de química fina em meio apolar. Empregando-se benzaldeído e cianoacetato de etila como reagentes, à temperatura ambiente, o catalisador $\mathrm{CTA}^{+}-[\mathrm{Si}]-$ MCM-41 resultou em $82 \%$ de conversão em apenas $1 \mathrm{~h}$ de reação $0^{44}$. Segundo os autores, a basicidade desse material localiza-se nos sítios aniônicos $\mathrm{SiO}^{-}$localizados na entrada dos poros.

\section{Alquilação do tolueno}

A alquilação lateral do tolueno, além de ser uma reação modelo que fornece bons resultados sobre as propriedades ácido-base dos catalisadores, desperta interesse devido ao fato de que o estireno, produto resultante da reação com metanol, na presença de um catalisador básico, tem grande demanda na produção industrial do termoplástico poliestireno ${ }^{74}$.

Sobre catalisadores ácidos, a alquilação de aromáticos ocorre em posição orto ou para do anel benzênico, enquanto que sobre catalisadores básicos a alquilação ocorre na cadeia lateral do tolueno (grupo metila). Quando se emprega zeólita faujasita trocada com césio como catalisador, o tolueno sofre alquilação lateral com metanol, produzindo estireno ${ }^{58,70}$. Nesta reação o metanol é desidrogenado sobre os sítios básicos a formaldeído (Equação 9), que é o reagente responsável pela alquilação do grupo metila do tolueno (Equação 10). Na Equação 11 o estireno é hidrogenado a etilbenzeno com o $\mathrm{H}_{2}$ produzido na Equação 10. Esta reação, portanto, gera uma mistura de etilbenzeno e estireno. Os sítios básicos do catalisador promovem a abstração de um próton do grupo metilênico do tolueno formando um intermediário carbânium ${ }^{71}$.

$\mathrm{CH}_{3} \mathrm{OH} \rightarrow \mathrm{HCHO}+\mathrm{H}_{2}$
$\mathrm{C}_{6} \mathrm{H}_{5} \mathrm{CH}_{3}+\mathrm{HCHO} \rightarrow \mathrm{C}_{6} \mathrm{H}_{5} \mathrm{CH}=\mathrm{CH}_{2}+\mathrm{H}_{2} \mathrm{O}$

$\mathrm{C}_{6} \mathrm{H}_{5} \mathrm{CH}=\mathrm{CH}_{2}+\mathrm{H}_{2} \rightarrow \mathrm{C}_{6} \mathrm{H}_{5} \mathrm{CH}_{2} \mathrm{CH}_{3}$

Uma reaçãoentre as reações muito estudadas na avaliação da basicidade e da acidez de catalisadores sólidos, é a desidrogenaçãodesidratação de álcoois. Quando se emprega isopropanol ${ }^{4}$, os produtos formados, acetona e propeno, provêm exclusivamente da reação de desidrogenação e desidratação, respectivamente, sendo que os sítios básicos são responsáveis pela primeira e os sítios ácidos, pela segunda reação. Na desidrogenação do isopropanol (Equação 12) a $425{ }^{\circ} \mathrm{C}$ Hathaway e Davis ${ }^{30}$ verificaram que, para uma mesma conversão, a seletividade à acetona aumenta com a basicidade dos catalisadores empregados, ou seja, $\mathrm{MgO}>\mathrm{Cs}-\mathrm{FAU}_{\text {impregnada }}>\mathrm{Cs}-$ $\mathrm{FAU}_{\text {trocada }}$. Em um outro trabalho ${ }^{71}$, envolvendo a alquilação do tolueno com metanol, os mesmos autores observaram que, apesar da maior atividade do óxido de magnésio na reação de desidrogenação, não ocorreu alquilação lateral do tolueno. Este fato mostra que outros fatores, além da presença de sítios básicos, são importantes na alquilação do tolueno. Na reação de desidrogenação, a maior basicidade do catalisador $\mathrm{MgO}$ facilita a abstração protônica na formação do intermediário $\mathrm{H}_{3} \mathrm{C}\left(\mathrm{CO}^{-}\right) \mathrm{CH}_{3}$, aumentando sua atividade.

$\mathrm{H}_{3} \mathrm{C}(\mathrm{COH}) \mathrm{CH}_{3} \rightarrow \mathrm{H}_{3}(\mathrm{C}=\mathrm{O}) \mathrm{CH}_{3}+\mathrm{H}_{2}$

Por que as zeólitas são mais ativas que o $\mathrm{MgO}$, na alquilação da cadeia lateral do tolueno? Há dois possíveis fatores para explicar este comportamento ${ }^{69}$. O primeiro está relacionado com os sítios ácidos $\mathrm{M}^{+}$das zeólitas que, ao interagirem com formaldeído inibem sua decomposição a $\mathrm{CO}$ e $\mathrm{H}_{2}$. $\mathrm{O}$ segundo fator, sugerido por cálculos quânticos ${ }^{67}$, está relacionado com o estado de transição bimolecular (Figura 7). Nesse modelo, o grupo metila do tolueno é adsorvido em um sítio básico da zeólita e o anel aromático interage com um sítio ácido. O formaldeído, por outro lado, é ativado por um sítio ácido em uma posição oposta ao complexo tolueno/zeólita. Na apresentação da Figura 7, a distância entre o par ácido-base, que ativa o tolueno, e o sítio ácido, que ativa o intermediário formaldeído, está em torno de 0,6-0,7 nm, correspondendo aproximadamente às dimensões dos poros da zeólita faujasita (Tabela 1). A importância desta configuração particular dos sítios ácidos e básicos, que é fornecida pela zeólita faujasita, explica a maior atividade das faujasitas trocadas com cátions alcalinos, em comparação aos outros catalisadores não zeolíticos ${ }^{72}$.

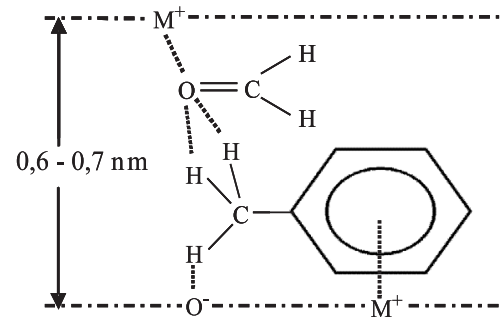

Figura 7. Configuração de sítios ácidos e básicos. Adaptada da ref. 69

\section{CONSIDERAÇÕES FINAIS}

Os métodos empregados na preparação de peneiras moleculares, com propriedades básicas para aplicação em catálise, foram apresentados e discutidos. Apesar da grande variedade de catalisadores obtidos pelos diferentes métodos, somente uma pequena parte deles é empregada atualmente em reações orgânicas. Há duas razões que fazem com que o uso de catalisadores básicos ainda seja pe- 
queno: a rápida adsorção do $\mathrm{CO}_{2}$ atmosférico, quando se empregam óxidos metálicos de alta basicidade, o que pode levar a uma desativação, exigindo tratamento in situ a uma alta temperatura e, os processos petroquímicos, que são os grandes consumidores de catalisadores industriais, empregam reações que necessitam principalmente de catalisadores ácidos.

Algumas reações orgânicas se processam somente via catálise básica. $\mathrm{O}$ estudo de peneiras moleculares com propriedades básicas, particularmente aquelas com sítios básicos fortes, ainda é recente. A fim de revelar o potencial de aplicação destes materiais, já crescente na química fina, é necessário que suas propriedades sejam melhor conhecidas e que um número maior de reações seja avaliado.

\section{AGRADECIMENTOS}

L. Martins agradece à FAPESP pela concessão de bolsa de Doutorado (processo 04/10762-6).

\section{REFERÊNCIAS}

1. Weitkamp, J.; Hunger, M.; Rymsa, U.; Microporous Mesoporous Mater. 2001, 48, 255.

2. Tanabe, K.; Hölderich, W. F.; Appl. Catal., A 1999, 181, 399.

3. Dartt, C. B.; Davis, M. E.; Catal. Today 1994, 19, 151.

4. Monteiro, J. F.; Veloso, C. O.; Actas del XIII Congresso Argentino de Catálise $y$ del $2^{\circ}$ Congresso de Catálise do Mercosul, Córdoba, Argentina, 2003.

5. Mitchell, P. W. D.; U. S. pat. 4,874,900 1989.

6. Sanseverino, A. M.; Quim. Nova 2000, 23, 102.

7. Nagy, J. B.; Bodart, P.; Hannus, I.; Kiricsi, I.; Synthesis, Characterization and Use of Zeolitic Microporous Materials, $1^{\text {st }}$ ed., DecaGen: Hungary, 1998.

8. Luna, F. J.; Schuchardt, U.; Quim. Nova 2001, 24, 885

9. Tu, M.; Davis, R. J.; J. Catal. 2001, 199, 85.

10. Davis, R. J.; J. Catal. 2003, 216, 396.

11. Martins, L.; Cardoso, D.; Quim. Nova 2005, 28, 264.

12. Beck, J. S.; Chu, C.; Johnson, Y.; Kresge, C. T.; Leonowicz, M. E.; Roth, W.; Vartuli, J. C.; pat. W.O. 113901991.

13. Beck, J. S.; Vartuli, J. C.; Roth, W. J.; Leonowicz, M. E.; Kresge, C. T.; Schmitt, K. D.; Chu, C. T.-W.; Olson, D. H.; Sheppard, E. W.; McCullen, S. B.; Higgins, J. B.; Schlenker, J. L.; J. Am. Chem. Soc. 1992, 114, 10834.

14. Barthomeuf, D.; Microporous Mesoporous Mater. 2003, 66, 1.

15. Wallau, M.; Schuchardt, U.; J. Braz. Chem. Soc. 1995, 6, 393.

16. Barthomeuf, D.; J. Phys. Chem. 1984, 88, 42.

17. Joshi, U. D.; Joshi, P. N.; Tamhankar, S. S.; Joshi, V. V.; Rode, C. V.; Shiralkar, V. P.; Appl. Catal., A 2003, 239, 209.

18. Mortier, W. J.; J. Catal. 1978, 55, 138.

19. Barthomeuf, D.; Stud. Surf. Sci. Catal. 1991, 65, 157.

20. Tsou, J.; Magnoux, P.; Guisnet, M.; Órfão, J. J. M.; Figueiredo, J. L.; Appl. Catal., B 2004, 51, 129.

21. Dzwigaj, S.; Mallmann, A.; Barthomeuf, D.; J. Chem. Soc., Faraday Trans. 1990, 86, 431 .

22. Barthomeuf, D.; Catal. Rev. Sci. Eng. 1996, 38, 521.

23. Barth, J. O.; Schenkel, R.; Kornatowski, J.; Lercher, J. A.; Stud. Surf. Sci. Catal. 2001, 135, 136.

24. Vayssilov, G. N.; Rösch, N.; J. Catal. 1999, 186, 423.

25. Wichterlová, B.; Dědeček, J.; Sobalík, Z.; Čejka, J.; Stud. Surf. Sci. Catal. 2001, 135, 344.

26. Čejka, J.; Dědeček, J.; Kotrla, J.; Tudor, M.; Ilková, N.; Ernst, S.; Stud. Surf. Sci. Catal. 2001, 135, 352.

27. Rice, M. J.; Chakraborty, A. K.; Bell, A. T.; J. Catal. 1999, 186, 222.

28. Guisnet, M.; Ribeiro, F. R.; Zeólitos: Um Nanomundo a Serviço da Catálise, $1^{\mathrm{a}}$ ed., Fundação Calauste Gulbenkian: Lisboa, 2004.

29. Martens, L. R. M.; Grobet, P. J.; Jacobs, P. A.; Nature 1985, 315, 568.

30. Hathaway, P. E.; Davis, M. E.; J. Catal. 1989, 116, 263.

31. Veloso, C. O.; Pinto, A. C.; Santos, E. N.; Monteiro, J. L. F.; Stud. Surf. Sci. Catal. 2001, 135, 146.

32. Volcho, K.; Kurbakova, S.; Kochagina, D.; Suslov, E.; Salakhutdinov, N.; Toktarev, A.; Echevskii, G.; Barkhash, V.; J. Mol. Catal. A: Chem. 2003, 195, 263.

33. On, D. T.; Desplantier-Giscard, D.; Danumah, C.; Kaliaguine, S.; Appl. Catal., A 2001, 222, 299.
34. Pinnavaia, T. J.; Kim, S.-S.; Zhang, Z.; Liu, Y.; Stud. Surf. Sci. Catal. 2004, $154,14$.

35. Tanev, P. T.; Chibwe, M.; Pinnavaia, T. J.; Nature 1994, 368, 321.

36. Zhang, W.; Froeba, M.; Wang, J.; Tanev, P. T.; Wong, J.; Pinnavaia, T. J.; J. Am. Chem. Soc. 1996, 118, 9164.

37. Zhao, D.; Huo, Q.; Feng, J.; Chmelka, B. F.; Stucky, G. D.; J. Am. Chem. Soc. 1998, 120, 6024.

38. Bonelli, B.; Ribeiro, M. F.; Antunes, A. P.; Valange S.; Gabelica, Z.; Garrone, E.; Microporous Mesoporous Mater. 2002, 54, 305.

39. Kloetstra, K. R.; van Laren, M.; van Bekkum, H.; J. Chem. Soc., Faraday Trans. 1997, 93, 1211.

40. Pérez, C. N.; Moreno, E.; Henriques, C. A.; Valange, S.; Gabelica, Z.; Monteiro, J. L. F.; Microporous Mesoporous Mater. 2000, 41, 137.

41. Wei, Y. L.; Cao, Y.; Zhu, J. H.; Stud. Surf. Sci. Catal. 2004, 154, 878.

42. Kubota, Y.; Nishizaki, Y.; Sugi, Y.; Chem. Lett. 2000, 29, 998.

43. Kubota, Y.; Nishizaki, Y.; Ikeya, H.; Nagaya, J.; Sugi, Y.; Stud. Surf. Sci. Catal. 2002, 141, 553.

44. Kubota, Y.; Nishizaki, Y.; Ikeya, H.; Saeki, M.; Hida, T.; Kawazu, S.; Yoshida, M.; Fujii, H.; Sugi, Y.; Microporous Mesoporous Mater. 2004, 70, 135.

45. Yang, C.-M.; Chao, K.-J.; J. Chin. Chem. Soc. 2002, 49, 883

46. Brunel, D.; Cauvel, A.; Fajula, F.; Di Renzo, F.; Stud. Surf. Sci. Catal. 1995 97, 173

47. Fajula, F.; Brunel, D.; Microporous Mesoporous Mater. 2001, 48, 119.

48. Zhao, X. S.; Lu, G. Q.; J. Phys. Chem. B 1998, 102, 1556.

49. Mercier, L.; Pinnavaia, T. J.; Environ. Sci. Technol. 1998, 32, 2749.

50. Kimura, T.; Saeki, S.; Sugahara, Y.; Kuroda, K.; Langmuir 1999, 15, 2794.

51. Chen, S. C.; Kawi, S.; Langmuir 2002, 18, 4720.

52. Chatterjee, M.; Iwasaki, T.; Hayashi, H.; Onodera, Y.; Ebina, T.; Nagase, T.; Catal. Lett. 1998, 52, 21.

53. Cesteros, Y.; Haller, G. L.; Microporous Mesoporous Mater. 2001, 43, 171.

54. Aguado, J.; Serrano, D. P.; Escola, J. M.; Microporous Mesoporous Mater. 2000, 34, 43.

55. Lang, N.; Tuel, A.; Chem. Mater. 2004, 16, 2969.

56. Lang, N.; Tuel, A.; Microporous Mesoporous Mater. 2005, 77, 147.

57. Kloetstra, K. R.; van Bekkum, H.; Stud. Surf. Sci. Catal. 1997, 105, 431.

58. Hattori, H.; Chem. Rev. 1995, 95, 537.

59. Monteiro, J. L. F.; Veloso, C. O. Em $2^{\circ}$ Curso Ibero-americano sobre Caracterização de Catalisadores e Adsorventes; Cardoso, D.; Jordão, M. H.; Machado, F., eds.; 2001.

60. Veloso, C. O.; Pinto, A. C.; Monteiro, J. L. F.; Mota, C. A.; Martins, R. L.; Anais do $10^{\circ}$ Congresso Brasileiro de Catálise, Salvador, Brasil, 1999; Huang, M.; Adnot, A.; Kaliaguine, S.; J. Am. Chem. Soc. 1992, 114, 10005; Yagi, F.; Tsuji, H.; Hattori, H.; Microp Mat. 1997, 9, 237; Lavalley, J. C.; Catal. Today 1996, 27, 377; Lima, E.; Laspéras, M.; Ménorval, L.-C.; Tichit, D.; Fajula, F.; J. Catal. 2004, 223, 28; Stöcker, M.; Microp. Mat. 1996, 6, 235; Sánchez-Sánchez, M.; Vidal-Moya, J. A.; Blasco, T.; Stud. Surf. Sci. Catal. 2004, 154, 1769.

61. Take, J.-I.; Kikuchi, N.; Yoneda, Y.; J. Catal. 1971, 21, 164.

62. Veloso, C. O.; Monteiro, J. L. F.; Souza-Aguiar, E. F.; Stud. Surf. Sci. Catal. 1994, 84, 1913.

63. Aramendía, M. A.; Boráu, V.; García, I. M.; Jiménez, C.; Marinas, A.; Marinas, J. M.; Porras, A.; Urbano, F. J.; Appl. Catal., A 1999, 184, 115.

64. Zhang, G.; Hattori, H.; Tanabe, K.; Appl. Catal. 1988, 40, 183.

65. Yashima, T.; Suzuki, H.; Hara, N.; J. Catal. 1974, 33, 486.

66. Philippou, A.; Anderson, M. W.; J. Catal. 2000, 189, 395.

67. Corma, A.; Fornés, V.; Martín-Aranda, R. M.; García, H.; Primo, J.; Appl. Catal. 1990, 59, 237.

68. Goa, Y.; Wu, P.; Tatsumi, T.; J. Catal. 2004, 224, 107; Linares, C. F.; Goldwasser, M. R.; Machado, F. J.; Rivera, A.; Rodríguez-Fuentes, G.; Barrault, J.; Microporous Mesoporous Mater. 2000, 41, 69; Ren, Z.; Cao, W.; Tong, W.; Jing, X.; Synthetic Commun. 2002, 32, 1947; Siebenhaar, B.; Casagrande, B.; Studer, M.; Blaser, H.-U.; Can. J. Chem. 2001, 79, 566; Zhang, X.; Lai, E. S. M.; Martin-Aranda, R.; Yeung, K. L.; Appl. Catal., A 2003, 261, 109 .

69. Itoh, H.; Miyamoto, A.; Murakami, Y.; J. Catal. 1980, 64, 284.

70. Yashima, T.; Sato, K.; Hayasaka, T.; Hara, N.; J. Catal. 1972, 26, 303.

71. Hathaway, P. E.; Davis, M. E.; J. Catal. 1989, 119, 497.

72. Engelhardt, J.; Szany, J.; Valyon, J.; J. Catal. 1987, 107, 296

73. Siegel, H.; Eggersdorfer, M. Em Ulmann's Encyclopedia of Industrial Chemistry; Elvers, B.; Rounsaville, J. F.; Schulz, G., eds.; $5^{\mathrm{a}}$ ed., VCH: Germany, 1985, vol. A15.

74. James, D. H.; Castor, W. M. Em ref. 73, vol. A25. 